\title{
The Role of PD-L1 Expression and Intratumoral Lymphocytes in Response to Perioperative Chemotherapy for Urothelial Carcinoma
}

F. Erlmeier ${ }^{\mathrm{a}}$, A.K. Seitz ${ }^{\mathrm{b}}$, G. Hatzichristodoulou ${ }^{\mathrm{b}}$, L. Stecher ${ }^{\mathrm{c}}$, M. Retz ${ }^{\mathrm{b}}$, J.E. Gschwend ${ }^{\mathrm{b}}$, W. Weichert ${ }^{\mathrm{a}, \mathrm{d}}$, H.R. Kübler ${ }^{\mathrm{b}}$ and T. Horn ${ }^{\mathrm{b}, *}$

${ }^{a}$ Institute for Pathology and Pathological Anatomy, Technische Universität München, Munich, Germany

${ }^{\mathrm{b}}$ Department of Urology, Klinikum rechts der Isar, Technische Universität München, Munich, Germany

${ }^{\mathrm{c}}$ Institute for Medical Statistics and Epidemiology, Technische Universität München, Munich, Germany

${ }^{\mathrm{d}}$ Member of the German Cancer Consortium (DKTK)

\begin{abstract}
.
Introduction: Immunological pathways are relevant for the effectiveness of conventional cytotoxic chemotherapy. Recently, checkpoint inhibition of the PD-1/PD-L1 axis has been shown to be therapeutically relevant in urothelial carcinoma.

Objective: To monitor PD-L1 expression on tumor cells and intratumoral infiltration with CD8 positive lymphocytes during perioperative chemotherapy for urothelial cancer and to evaluate their use as potential predictive markers for chemotherapy. Patients and Methods: Sixty-four patients with muscle-invasive urothelial cancer were included in the analysis. Twenty-two patients received preoperative chemotherapy and 42 were treated in an adjuvant setting for locally advanced disease or lymph node metastases. PD-L1 status and the density of infiltration with CD8-positive cells were assessed by immunohistochemistry and analysed for their association with survival (adjuvant group) and response to chemotherapy (preoperative group). For PD-L1 positivity we used a cutoff of $10 \%$ positive tumor cells.

Results: In the adjuvant group, 11 of 42 patients (26.2\%) had PD-L1 positive tumor cells. Twenty-six of 42 (61.9\%) patients were highly infiltrated with CD8 + lymphocytes. There was no significant evidence of an association with overall survival for PD-L1 status nor for CD8 infiltration density ( $p=0.63$ and 0.71 ). In the preoperative group, eight of the $22(36.4 \%)$ patients were PD-L1 positive and 13 (59\%) were highly infiltrated with CD8 + lymphocytes before chemotherapy. There was no evidence of associations with response or survival. Eight patients showed a pathological response to preoperative treatment. These had a significantly longer overall survival than non-responders $(p=0.01)$. In the preoperative group the pre-treatment expression of the immunologic markers could be compared to the post-treatment status. Only one patient showed a changed PD-L1 status and three patients a changed CD8 status.

Conclusions: The tumoral expression of PD-L1 in urothelial carcinoma does not seem to be largely influenced by chemotherapy. Our data do not provide evidence that tumoral expression of PD-L1 and CD8 are useful as prognostic or predictive markers. Small sample size is the major limitation of our study.
\end{abstract}

Keywords: PD-L1, perioperative chemotherapy, bladder cancer

${ }^{*}$ Correspondence to: Thomas Horn, Department of Urology, Klinikum rechts der Isar, Ismaningerstraße 22, 81675 Munich, Germany. Tel.: +49 894140 2522; E-mail: t.horn@tum.de. 


\section{INTRODUCTION}

Urothelial carcinoma is an aggressive tumor with a poor prognosis in advanced stages [1]. Perioperative chemotherapy in addition to radical cystectomy is able to prolong survival [2]. While preoperative chemotherapy is anchored in guideline recommendations, a recent metaanalysis could show a statistically significant survival benefit also for adjuvant treatment [3]. This metaanalysis did not include another recently published trial which could confirm the benefit of adjuvant treatment in patients with locally advanced, or lymph node-positive disease compared to treatment on progression in terms of prolonging progression-free survival. As this study recruited slowly and thus was prematurely closed it was unable to show a prolongation of overall survival [4]. Up to now, there are no markers that can predict the success of perioperative chemotherapy in an individual patient.

The relevance of inflammatory tumor-host interactions for effective conventional chemotherapeutic treatment is widely accepted [5]. Moreover, the specific pharmacological inhibition of immune checkpoints that limit $\mathrm{T}$ cell function like the PD1/PD-L1 axis or LAG-3 and its combination with conventional chemotherapy is gaining attention in the treatment of cancer. Concerning urothelial carcinoma the inhibition of the immunologic checkpoint PD-1/PD-L1 has recently been shown to be therapeutically relevant [6]. This inhibition aims at restoring preexistent $\mathrm{T}$ cell responses which have been shown to exist in bladder cancer [7].

Several groups have assessed the prognostic ability of PD-L1 expression in urothelial cancer and described the expression of PD-L1 on tumor cells to be associated with a shorter survival after cystectomy [8-10]. Further, a recent publication was unable to confirm this association and described PD-L1 expression on tumor-infiltrating lymphocytes as a marker for improved outcome [11]. The association of the expression of these immune checkpoint molecules with the outcome of perioperative chemotherapy has not been evaluated so far. In other cancer entities this approach is promising: preoperative treatment of breast cancer was reported to be most efficacious if there is either a preexisting or a chemotherapy-induced antitumoral immune response [12]. In colorectal cancer, the success of chemotherapy is determined by the infiltration density with lymphocytes [13]. Likewise, in bladder cancer a high expression of immune-relevant genes seems to be associated with response to chemotherapy [14].

In our study, we analysed two distinct groups of patients. Firstly, patients with adjuvant treatment after radical cystectomy. For this group we had a definitive pre-chemotherapy histology as well as outcome data available, but no post-chemotherapy tissue to determine changes in immune status and no target lesion to monitor clinical response. In this group, we aimed at correlating PD-L1 status of the tumor and the infiltration density with CD8-positive cells with overall survival. The underlying hypothesis was that patients with a higher PD-L1 expression have a better response to adjuvant treatment and thus better outcome. Secondly, patients with preoperative chemotherapy for whom data on clinical and pathological response as well as outcome data were available. In this patient population, comparison of the immune status between pre- and posttreatment tumor tissue was possible. In this cohort, we primarily wanted to assess whether the PD-L1 status and the CD8 infiltration density is stable from pre- to postchemotherapy. Secondly, we conducted an analysis regarding response and survival.

\section{PATIENTS AND METHODS}

\section{Patients}

The data used in this study came from a prospectively kept database of all patients with urothelial carcinoma treated with perioperative chemotherapy at our tertiary care centre (approved by the local ethics committee, number 5292/12). Patients with adjuvant chemotherapy were included, if the tissue of the cystectomy could be obtained for pathological analysis. Indications for adjuvant treatment included locally advanced disease (pT3/pT4) or lymph node positivity (pN1-pN3). These two groups share a high risk of disease recurrence. Patient characteristics are shown in Table 1.

Patients with preoperative chemotherapy were included if pretreatment tissue was available. The indication for preoperative treatment of a histologically proven muscle-invasive cancer was determined by CT staging and included either signs of a locally advanced disease with infiltration of the perivesical fatty tissue or a metastatic involvement of lymph nodes. Moreover, hydronephrosis was considered 
Table 1

Patients with adjuvant chemotherapy. Follow-up duration is given in months

\begin{tabular}{|c|c|c|c|c|c|}
\hline $\begin{array}{l}\text { PD-L1 } \\
\text { Status }\end{array}$ & CD8 & Dead & $\begin{array}{c}\text { Duration } \\
\text { F/U }\end{array}$ & $\begin{array}{c}\mathrm{pT} \\
\text { Stage }\end{array}$ & $\begin{array}{c}\mathrm{pN} \\
\text { Stage }\end{array}$ \\
\hline negative & low & No & 17 & $3 a$ & $\mathrm{~N}+$ \\
\hline positive & high & No & 19 & $4 a$ & 0 \\
\hline positive & high & No & 19 & $3 b$ & 0 \\
\hline negative & high & No & 22 & $4 a$ & $\mathrm{~N}+$ \\
\hline negative & high & Yes & 26 & $3 b$ & $\mathrm{~N}+$ \\
\hline negative & low & Yes & 30 & $3 b$ & $\mathrm{~N}+$ \\
\hline negative & high & No & 40 & $3 b$ & $\mathrm{~N}+$ \\
\hline positive & high & Yes & 4 & $2 b$ & $\mathrm{~N}+$ \\
\hline negative & high & Yes & 12 & $3 a$ & 0 \\
\hline positive & low & Yes & 41 & $3 b$ & 0 \\
\hline negative & high & No & 48 & $3 a$ & $\mathrm{~N}+$ \\
\hline positive & high & Yes & 24 & $3 b$ & $\mathrm{~N}+$ \\
\hline positive & high & Yes & 18 & $2 a$ & $\mathrm{~N}+$ \\
\hline negative & high & Yes & 24 & $2 a$ & $\mathrm{~N}+$ \\
\hline positive & high & No & 61 & $3 b$ & $\mathrm{~N}+$ \\
\hline positive & high & No & 66 & $4 a$ & 0 \\
\hline negative & high & No & 73 & $3 a$ & 0 \\
\hline negative & high & No & 87 & 1 & $\mathrm{~N}+$ \\
\hline negative & low & Yes & 14 & $4 a$ & $\mathrm{~N}+$ \\
\hline positive & high & No & 66 & $2 a$ & $\mathrm{~N}+$ \\
\hline positive & high & No & 54 & $3 a$ & $\mathrm{~N}+$ \\
\hline negative & low & Yes & 41 & $2 b$ & $\mathrm{~N}+$ \\
\hline negative & low & No & 52 & $3 b$ & $\mathrm{~N}+$ \\
\hline negative & high & Yes & 22 & $3 b$ & $\mathrm{~N}+$ \\
\hline negative & high & Yes & 35 & $4 a$ & $\mathrm{~N}+$ \\
\hline negative & high & Yes & 8 & $4 a$ & 0 \\
\hline positive & high & Yes & 31 & $3 b$ & 0 \\
\hline negative & low & No & 65 & 1 & $\mathrm{~N}+$ \\
\hline negative & low & No & 43 & $3 a$ & 0 \\
\hline negative & high & No & 67 & $2 b$ & $\mathrm{~N}+$ \\
\hline negative & low & Yes & 42 & 1 & $\mathrm{~N}+$ \\
\hline negative & high & Yes & 67 & $3 b$ & 0 \\
\hline negative & low & No & 81 & $3 b$ & $\mathrm{~N}+$ \\
\hline negative & low & No & 78 & $3 b$ & 0 \\
\hline negative & low & Yes & 44 & $3 b$ & $\mathrm{~N}+$ \\
\hline negative & low & Yes & 36 & 3 & $\mathrm{~N}+$ \\
\hline negative & low & No & 91 & 4 & 0 \\
\hline negative & low & No & 99 & $3 b$ & $\mathrm{~N}+$ \\
\hline negative & high & No & 52 & $3 b$ & 0 \\
\hline negative & low & Yes & 46 & $4 a$ & $\mathrm{~N}+$ \\
\hline negative & high & No & 109 & $2 a$ & $\mathrm{~N}+$ \\
\hline negative & high & Yes & 42 & $2 a$ & $\mathrm{~N}+$ \\
\hline
\end{tabular}

a sign of locally advanced disease, if it was present prior to transurethral tumor resection [15]. Pathological response was defined as the lack of both muscle-invasive residual disease and lymph node metastases at the time of cystectomy. Patient characteristics are shown in Table 2. Two patients had urothelial carcinoma of the upper urinary tract, all others of the urinary bladder.

Chemotherapy was given with a platinum-based combination therapy. Mostly the combination of gemcitabine and cisplatin was used. In three patients cisplatin was replaced by carboplatin due to renal insufficiency.

This specific analysis of the database was approved by the local ethics committee (number 147/15).

\section{Methods}

Immunohistochemistry was conducted on archived, formalin-fixed, paraffin-embedded (FFPE) tissue. All tissue sections were reviewed for confirmation of the original diagnosis by a pathologist (FE) and staged according to the 2010 American Joint Committee on Cancer TNM Classification. Standard immunohistochemistry analysis was performed for PD-L1 using a murine antihuman monoclonal antibody (PD-L1, XP Rabbit mAb, Cell Signaling, \#1368) at a dilution of 1:200 according to standard automated methods with a Benchmark XT slide stainer. Human placenta was used as a positive control. Immunohistochemistry for CD8 was conducted according to standard automated methods with a Benchmark XT slide stainer according to the manufacturer's instructions and protocols.

Hematoxylin/eosin, PD-L1- and CD8-stained sections were reviewed by a pathologist (FE) blinded to clinical outcome. Besides TNM stage the presence of intratumoral lymphocytic infiltration and the quantity and location of PD-L1 staining were evaluated. The tumor was considered positive for PD-L1 if $10 \%$ of the tumor cells were positive for plasma membrane staining. Intratumoral CD8 $+\mathrm{T}$ cells were scored as "low" or "high" density with a cut point of approximately 60 cells per typical high power field (HPF). The cut point of 60 cells per HPF was already used in several studies in regard to urothelial carcinoma as well as other tumor entities [16-18]. Representative images are shown in Fig. 1.

\section{Statistical analysis}

Overall survival curves were estimated using Kaplan Meier analysis and compared between subgroups using log-rank tests. For the preoperative sample, chi-square tests were performed to assess the association between PD1 status and preoperative chemotherapy response, and CD8 status and response. $P$ values $<0.05$ were considered statistically significant. Statistical analyses were performed using the statistical software package $\mathrm{R}$ (version 3.1.3, the R Foundation for statistical computing). 
Table 2

Patients with neoadjuvant chemotherapy. N.a. = not available due to lack of tumor tissue in a pT0, pN0 situation. LA = locally advanced in CT staging. $\mathrm{LN}+=$ suspicious lymph nodes in preoperative staging. $*=$ Urothelial carcinoma of the upper urinary tract

\begin{tabular}{|c|c|c|c|c|c|c|}
\hline \multicolumn{2}{|c|}{ Preoperative } & \multicolumn{2}{|c|}{ Postoperative } & \multirow[t]{2}{*}{ Response } & \multirow[t]{2}{*}{ Histology } & \multirow[t]{2}{*}{ Indication } \\
\hline PD-L1-Tumor & $\mathrm{CD} 8$ & PD-L1-Tumor & $\mathrm{CD} 8$ & & & \\
\hline negative & high & n.a. & n.a. & yes & pT0, pN0 & LA \\
\hline negative & low & n.a. & n.a. & yes & pTis, pN0 & $\mathrm{LN}+/ \mathrm{LA}$ \\
\hline negative & high & n.a. & n.a. & yes & $\mathrm{pTa}, \mathrm{pN} 0$ & $\mathrm{LN}+$ \\
\hline negative & high & n.a. & n.a. & yes & pT0, pNo* & $\mathrm{LN}+/ \mathrm{LA}$ \\
\hline negative & low & n.a. & n.a. & yes & pT0, pNo & $\mathrm{LN}+/ \mathrm{LA}$ \\
\hline positive & high & negative & high & yes & $\mathrm{pT} 1, \mathrm{pN} 0$ & LA \\
\hline positive & high & n.a. & n.a. & yes & pT0, pNO & $\mathrm{LN}+$ \\
\hline positive & high & n.a. & n.a. & yes & pT0, pN0 & LA \\
\hline negative & high & negative & high & no & pT2b, pN0 & LA \\
\hline negative & low & negative & low & no & pT3a, pN0 & LA \\
\hline negative & low & negative & low & no & $\mathrm{pT} 3 \mathrm{~b}, \mathrm{pN} 3$ & LA \\
\hline negative & low & negative & low & no & pT0, pN1 & LA \\
\hline negative & low & negative & high & no & pT3a, pN0 & LA \\
\hline negative & low & negative & low & no & pT4b, pN1 & LA \\
\hline negative & high & negative & high & no & $\mathrm{pT} 3 \mathrm{~b}, \mathrm{pN} 1$ & LA \\
\hline negative & high & negative & low & no & pT3a, pN3 & $\mathrm{LN}+$ \\
\hline negative & low & negative & low & no & pT4a, pN3 & $\mathrm{LN}+/ \mathrm{LA}$ \\
\hline positive & high & positive & high & no & pT4a, pN3 & $\mathrm{LN}+/ \mathrm{LA}$ \\
\hline positive & low & $\mathrm{x}$ & $\mathrm{x}$ & no & $\mathrm{pT} 3 \mathrm{~b}, \mathrm{pN} 0$ & $\mathrm{LN}+/ \mathrm{LA}$ \\
\hline positive & high & positive & low & no & $\mathrm{pT} 3 \mathrm{~b}, \mathrm{pN} 3$ & $\mathrm{LN}+/ \mathrm{LA}$ \\
\hline positive & high & positive & high & no & pT3, pN1* & $\mathrm{LN}+/ \mathrm{LA}$ \\
\hline positive & high & positive & high & no & pT3b, pN2 & $\mathrm{LN}+/ \mathrm{LA}$ \\
\hline
\end{tabular}

\section{RESULTS}

\section{Patient cohort}

We identified 42 patients with adjuvant and 22 patients with preoperative treatment. The median age of the adjuvantly treated patients was 65 years including 35 male and seven female patients. The preoperative group had a median age of 67 years with 14 male and eight female patients. All patients were of Caucasian origin and all patients had conventional urothelial carcinoma. Other baseline data of both patient groups are shown in Tables 1 and 2. All patients received a platinum-containing combination chemotherapy. Mostly the combination of gemcitabine and cisplatin was used. Cisplatin was replaced by carboplatin in case of renal insufficiency. In the adjuvant setting, all patients received gemcitabine and cisplatin.

Twenty of 42 patients in the adjuvant group (47.6\%) and 10 of 22 patients $(45.5 \%)$ in the neoadjuvant group had died during follow-up. Median follow-up time of patients still alive was 63 months. Among 22 patients with preoperative treatment there were eight pathological responses $(36 \%)$ and five of these eight patients had a complete response.
Clinical and immunohistochemical data are summarized in Tables 1 (adjuvant) and 2 (preoperative).

In the adjuvant group, neither $\mathrm{pT}$ nor $\mathrm{pN}$ stage had a distinct influence on survival, as there was no significant evidence of differences in survival between patients with an locally limited (pT1/2, $\mathrm{pN}+, n=11)$ or a locally advanced (pT3/4, pNX, $n=31)$ tumor $(p=0.89)$. Also, there was no significant evidence of a difference in survival between patients with $\mathrm{pN}+(n=29)$ or $\mathrm{pN} 0(n=13)$ disease $(p=0.54)$.

In the preoperative group, 8 of 22 patients were responders. These, in comparison with nonresponders had improved overall survival rates $(p=0.01$, see Fig. 2).

\section{PD-L1 status persists over preoperative treatment}

Of the 22 patients with preoperative chemotherapy $14(63.6 \%)$ were tested negative for PD-L1 in tumor tissue and $8(36.4 \%)$ were tested positive. As postchemotherapy data were not available for patients with pT0, pTa or pTis tumors and for one patient the post-treatment tissue was missing, we were able to compare tissue of 14 patients from pre- to postchemotherapy. All five patients tested PD-L1 positive 
kept their positive status. Of nine PD-L1 negative patients only one was tested positive after chemotherapy. However, this single case had only a pT1 tumor after preoperative treatment, which may be difficult to judge immunohistochemically due to the paucity of invasive tumor tissue.

Concerning CD8 infiltration density five patients were tested "low" both before and after chemotherapy

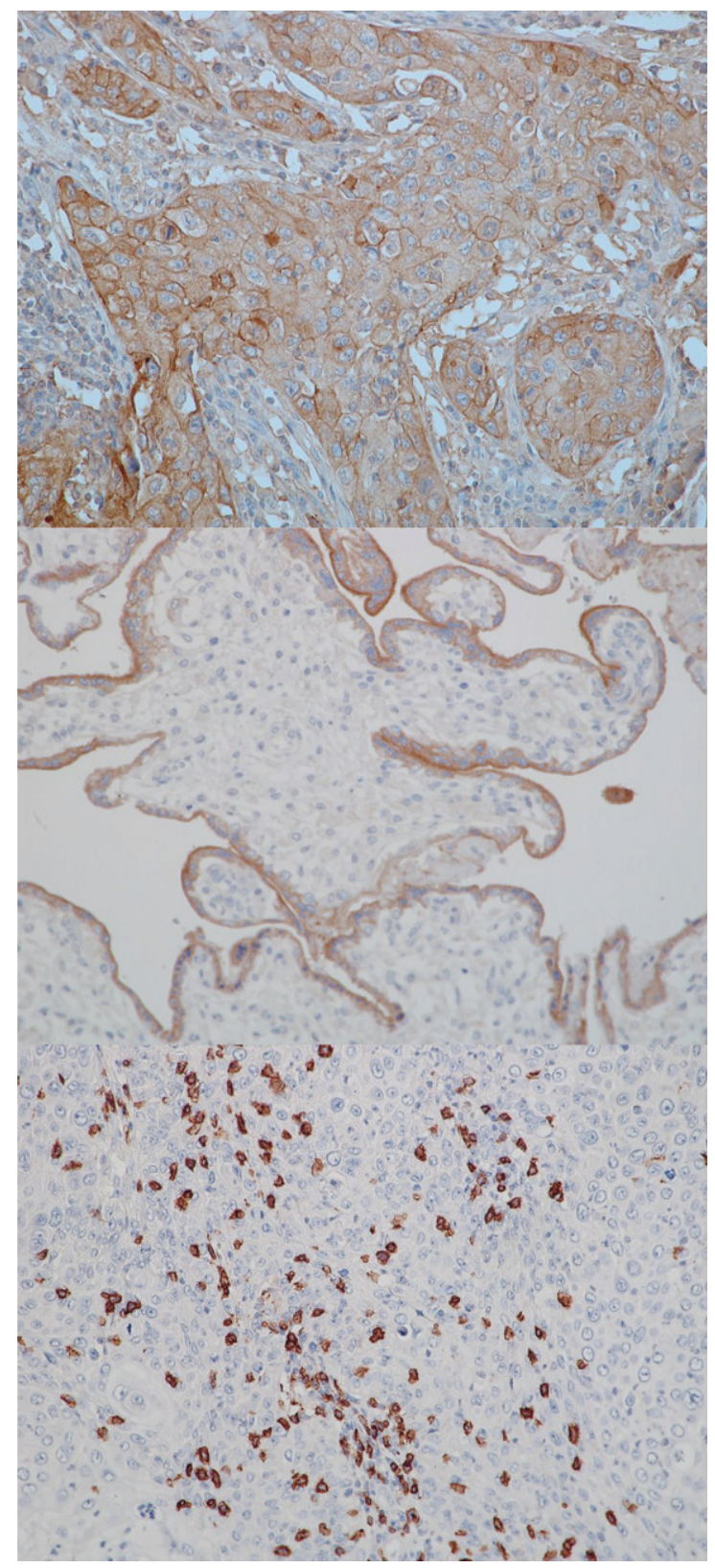

Fig. 1. Exemplary stains for PD-L1 and CD8. From top to bottom: PD-L1 positive tumor, 20x; positive control (human placenta) for PD-L1, 20x; CD8 infiltration, 20x. and six patients were consistently tested as "high". Three patients converted from "high" to "low" status during preoperative treatment.

\section{PD-L1 status seems unable to predict the response to chemotherapy}

In the adjuvant group, 31 patients were tested PDL1 negative and 11 patients PD-L1 positive. Fifteen of 31 patients tested negative and five of eleven patients tested positive died during the follow-up period. The Kaplan-Meier curve for overall survival is shown in Fig. 3. There was no significant evidence of a difference in survival between the two groups $(p=0.63)$.

Moreover, in the preoperative group PD-L1 status had no predictive power. Five of fourteen $(35.7 \%)$ negative patients and three of eight $(37.5 \%)$ positive patients showed responses. There was no significant evidence of a difference in overall survival conveyed by PD-L1 status ( $p=0.97$, Fig. 4).

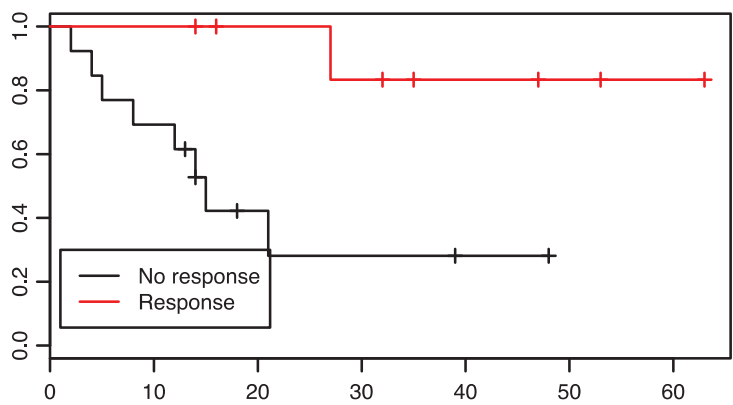

Fig. 2. Patients with a response to neoadjuvant treatment had a significantly prolonged overall survival in comparison to nonreponders, $p=0.01$.

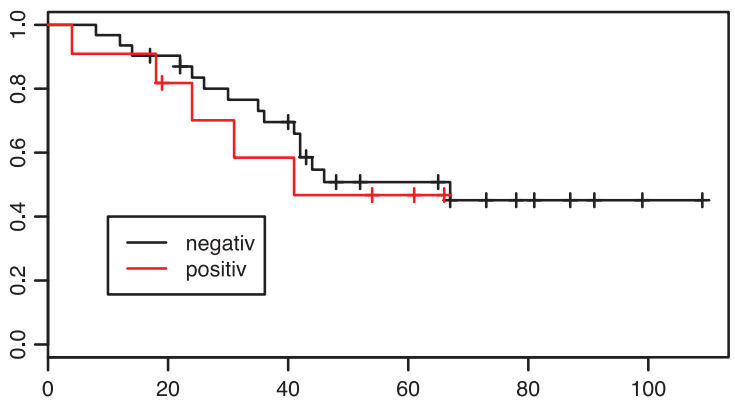

Fig. 3. Kaplan-Meier estimates of survival according to PD-L1 tumor status in the adjuvant group. $\mathrm{X}$ axis: Overall survival in months. $p=0.63$. 


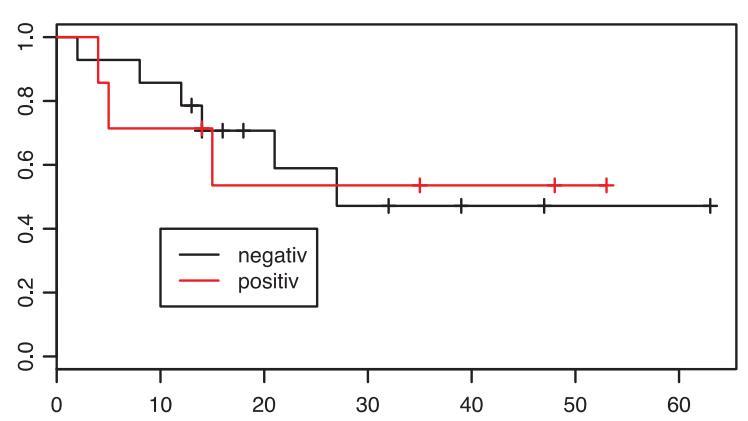

Fig. 4. Kaplan-Meier estimates of survival according to PD-L1 tumor status in the neoadjuvant group. $\mathrm{X}$ axis: Overall survival in months. $p=0.97$.

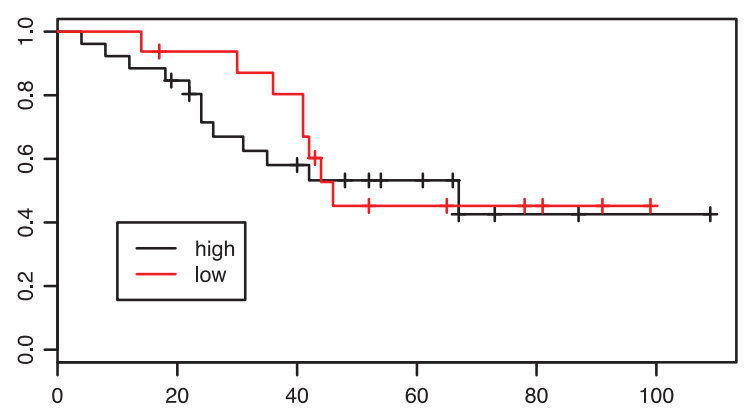

Fig. 5. Kaplan-Meier estimates of survival according to intratumoral CD8 infiltration density in the adjuvant group. $p=0.71$.

\section{High CD8 positivity is not associated with overall survival or the response to chemotherapy}

Of 42 patients in the adjuvant group 26 were classified as having "high" CD8 infiltration. Looking at the Kaplan-Meier curves these patients did not have improved overall survival in comparison with patients with low CD8 infiltration ( $p=0.71$, Fig. 5).

In the preoperative group 13 of 22 patients had a high CD8 infiltration before chemotherapy. There was no significant evidence of an association between CD8 status and response: six of 13 patients (46.2\%) in the group with high CD8 infiltration and 2/9 $(22 \%)$ patients in the group with low CD8 infiltration were responders $(p=0.49)$. Regarding overall survival there was also no difference between the CD8 "low" and "high" groups ( $p=0.61$, Fig. 6).

\section{DISCUSSION}

We analyzed a cohort of 64 patients with urothelial cancer and a perioperative chemotherapy for their tumoral expression of PD-L1 and their infiltration density with $\mathrm{CD} 8+$ lymphocytes. The results were

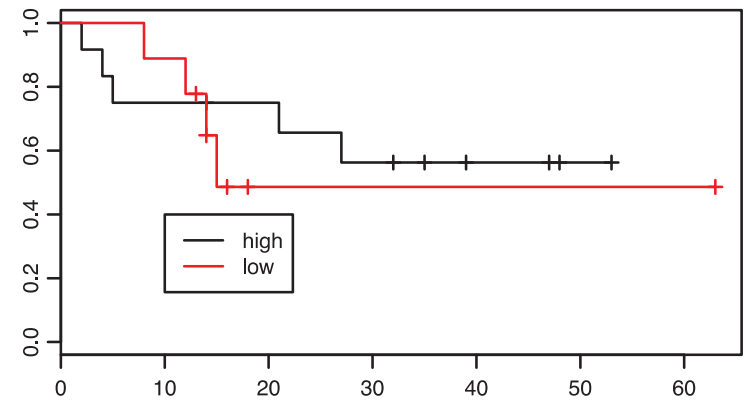

Fig. 6. Kaplan-Meier estimates of survival according to intratumoral CD8 infiltration density in the neoadjuvant group. $p=0.61$.

correlated with overall survival and the response to chemotherapy. The quest for predictive or prognostic markers for chemotherapeutic treatment of urothelial cancer is of utmost importance, as we know that response makes a significant prognostic difference for the patients [19], which we were able to confirm in our study.

In general, one needs to keep in mind that response and change in PD-L1 or CD8 status can only be assessed in the preoperative group, whereas survival analyses can be done in both groups

Our results suggest that the tumoral expression of PD-L1 is not strongly influenced by preoperative chemotherapy. This finding is relevant for clinical trials with inhibitors of the PD-1/PD-L1 interaction. In these trials patients are stratified by the expression of PD-L1 by their tumors and it remains a matter of debate whether this tissue needs to be obtained before any kind of chemotherapy. Our data support the conclusion that in most cases the timing of the retrieval of a tissue sample has low relevance in terms of tumoral PD-L1 expression.

In our study there was only one case with a divergent result from pre- to post-chemotherapy. This was a case with a posttreatment pathology of a pT1 tumor. In these cases with only minimal infiltration it may be difficult for the pathologist to correctly judge immunohistochemical parameters. On the other hand, this remaining pT1 tumor may not be representative for the previously proven muscleinvasive tumor. To our knowledge, this is the first study to look for changes in the PD-L1 expression pattern conveyed by surgery or cytotoxic therapy.

Also the density of intratumoral CD8+ lymphocytes remained a rather stable parameter during treatment. Only in three of 14 cases a change from "high" to "low" was seen. CD8 status remained stable in the remaining cases. No prior 
study has looked at changes in CD8 infiltration density during cytotoxic treatment.

Next, we wanted to assess for the ability of PD-L1 and CD8 to predict the response to chemotherapy and survival. Both markers have proven to be of prognostic value in other tumor entities like colorectal $[20,21]$ or renal cell carcinoma [22]. Usually, a higher CD8 infiltration as a surrogate marker for the extent of interaction between the host's immune system and tumor cells is associated with a better prognosis. PD-L1 expression on tumor cells rather masks and thereby diminuates existing antitumor immune responses and is associated with a poor prognosis. These markers seem not only relevant for the assessment of the patients' prognosis, but also for the prediction of the response to chemotherapy. For example, the ratio of CD8 and FOXP3 + lymphocytes was able to predict the success of chemotherapy in breast cancer [23, 24]. Also in breast cancer, patients with a high PD-L1 expression were more likely to have a pathologically complete response after preoperative treatment [25]. On the other hand, studies in lung cancer trying to establish PD-L1 as a predictive marker failed [26].

Concerning bladder cancer we, amongst others, had been able to show that a high CD8 infiltration in cystectomy specimens is correlated with a strong trend towards a better prognosis [27, 28], whereas conflicting studies were published regarding PD-L1 [8-11].

Our study did not provide evidence of an association between both CD8 and PD-L1 and survival after adjuvant or preoperative chemotherapy as well as pathological response to preoperative treatment.

In conclusion, tumoral PD-L1 is a stable marker, uninfluenced by preoperative chemotherapy in urothelial carcinoma. In our study, there was no association between pretreatment PD-L1 status or CD8 infiltration and both prognosis and response to chemotherapy. Nevertheless, these parameters may be important in guiding treatment decisions with PD1/PD-L1 inhibitors.

The main limitation of this study is its small sample size.

\section{CONFLICT OF INTEREST}

TH has been an employee of MSD Sharp and Dohme since September 1st 2016. Study planning, data generation and analysis as well as manuscript preparation were completed before August 31st 2016.

\section{REFERENCES}

[1] Stein JP, Lieskovsky G, Cote R, Groshen S, Feng AC, Boyd $\mathrm{S}$, et al. Radical cystectomy in the treatment of invasive bladder cancer: Long-term results in 1,054 patients. Journal of Clinical Oncology: Official Journal of the American Society of Clinical Oncology 2001;19(3):666-75.

[2] Grossman HB, Natale RB, Tangen CM, Speights VO, Vogelzang NJ, Trump DL, et al. Neoadjuvant chemotherapy plus cystectomy compared with cystectomy alone for locally advanced bladder cancer. N Engl J Med 2003;349(9): 859-66.

[3] Leow JJ, Martin-Doyle W, Rajagopal PS, Patel CG, Anderson EM, Rothman AT, et al. Adjuvant chemotherapy for invasive bladder cancer: A 2013 updated systematic review and meta-analysis of randomized trials. European Urology 2014;66(1):42-54.

[4] Sternberg CN, Skoneczna I, Kerst JM, Albers P, Fossa SD, Agerbaek M, et al. Immediate versus deferred chemotherapy after radical cystectomy in patients with pT3-pT4 or $N+\mathrm{M} 0$ urothelial carcinoma of the bladder (EORTC 30994): An intergroup, open-label, randomised phase 3 trial. The Lancet Oncology 2015;16(1):76-86.

[5] Emens LA, Middleton G. The interplay of immunotherapy and chemotherapy: Harnessing potential synergies. Cancer Immunology Research 2015;3(5):436-43.

[6] Powles T, Eder JP, Fine GD, Braiteh FS, Loriot Y, Cruz C, et al. MPDL3280A (anti-PD-L1) treatment leads to clinical activity in metastatic bladder cancer. Nature 2014; 515(7528):558-62.

[7] Horn T, Grab J, Schusdziarra J, Schmid S, Maurer T, Nawroth R, et al. Antitumor $\mathrm{T}$ cell responses in bladder cancer are directed against a limited set of antigens and are modulated by regulatory $\mathrm{T}$ cells and routine treatment approaches. Int J Cancer 2013;133(9):2145-56.

[8] Boorjian SA, Sheinin Y, Crispen PL, Farmer SA, Lohse CM, Kuntz SM, et al. T-cell coregulatory molecule expression in urothelial cell carcinoma: Clinicopathologic correlations and association with survival. Clinical Cancer Research: An Official Journal of the American Association for Cancer Research 2008;14(15):4800-8.

[9] Xylinas E, Robinson BD, Kluth LA, Volkmer BG, Hautmann R, Kufer R, et al. Association of T-cell co-regulatory protein expression with clinical outcomes following radical cystectomy for urothelial carcinoma of the bladder. Eur $\mathrm{J}$ Surg Oncol 2014;40(1):121-7.

[10] Nakanishi J, Wada Y, Matsumoto K, Azuma M, Kikuchi $\mathrm{K}$, Ueda S. Overexpression of B7-H1 (PD-L1) significantly associates with tumor grade and postoperative prognosis in human urothelial cancers. Cancer Immunology, Immunotherapy: CII 2007;56(8):1173-82.

[11] Bellmunt J, Mullane SA, Werner L, Fay AP, Callea M, Leow JJ, et al. Association of PD-L1 expression on tumorinfiltrating mononuclear cells and overall survival in patients with urothelial carcinoma. Annals of Oncology: Official Journal of the European Society for Medical Oncology/ESMO. 2015.

[12] Stoll G, Enot D, Mlecnik B, Galon J, Zitvogel L, Kroemer G. Immune-related gene signatures predict the outcome of neoadjuvant chemotherapy. Oncoimmunology 2014;3(1):e27884.

[13] Halama N, Michel S, Kloor M, Zoernig I, Benner A, Spille A, et al. Localization and density of immune cells in the invasive margin of human colorectal cancer liver metastases are 
prognostic for response to chemotherapy. Cancer Research 2011;71(17):5670-7.

[14] Choi W, Porten S, Kim S, Willis D, Plimack ER, HoffmanCensits J, et al. Identification of distinct basal and luminal subtypes of muscle-invasive bladder cancer with different sensitivities to frontline chemotherapy. Cancer Cell 2014;25(2):152-65.

[15] Bartsch GC, Kuefer R, Gschwend JE, de Petriconi R, Hautmann RE, Volkmer BG. Hydronephrosis as a prognostic marker in bladder cancer in a cystectomy-only series. European Urology 2007;51(3):690-7; discussion 7-8.

[16] Faraj SF, Munari E, Guner G, Taube J, Anders R, Hicks J, et al. Assessment of Tumoral PD-L1 Expression and Intratumoral CD8+T Cells in Urothelial Carcinoma. Urology 2015;85(3):703.e1-6.

[17] Lipson EJ, Vincent JG, Loyo M, Kagohara LT, Luber BS, Wang H, et al. PD-L1 expression in the Merkel cell carcinoma microenvironment: Association with inflammation, Merkel cell polyomavirus and overall survival. Cancer Immunology Research 2013;1(1):54-63.

[18] Paulson KG, Iyer JG, Tegeder AR, Thibodeau R, Schelter $\mathrm{J}$, Koba S, et al. Transcriptome-wide studies of merkel cell carcinoma and validation of intratumoral CD8+lymphocyte invasion as an independent predictor of survival. Journal of Clinical Oncology: Official Journal of the American Society of Clinical Oncology 2011;29(12):1539-46.

[19] Petrelli F, Coinu A, Cabiddu M, Ghilardi M, Vavassori I, Barni S. Correlation of pathologic complete response with survival after neoadjuvant chemotherapy in bladder cancer treated with cystectomy: A meta-analysis. European Urology 2014;65(2):350-7.

[20] Galon J, Costes A, Sanchez-Cabo F, Kirilovsky A, Mlecnik B, Lagorce-Pages C, et al. Type, density, and location of immune cells within human colorectal tumors predict clinical outcome. Science 2006;313(5795):1960-4.

[21] Mlecnik B, Tosolini M, Kirilovsky A, Berger A, Bindea $\mathrm{G}$, Meatchi $\mathrm{T}$, et al. Histopathologic-based prognostic factors of colorectal cancers are associated with the state of the local immune reaction. J Clin Oncol 2011;29(6): 610-8.

[22] Thompson RH, Kuntz SM, Leibovich BC, Dong H, Lohse CM, Webster WS, et al. Tumor B7-H1 is associated with poor prognosis in renal cell carcinoma patients with longterm follow-up. Cancer Research 2006;66(7):3381-5.

[23] Asano Y, Kashiwagi S, Goto W, Kurata K, Noda S, Takashima T, et al. Tumour-infiltrating CD8 to FOXP3 lymphocyte ratio in predicting treatment responses to neoadjuvant chemotherapy of aggressive breast cancer. The British Journal of Surgery 2016;103(7):845-54.

[24] Miyashita M, Sasano H, Tamaki K, Chan M, Hirakawa $\mathrm{H}$, Suzuki A, et al. Tumor-infiltrating CD8+and FOXP3+ lymphocytes in triple-negative breast cancer: Its correlation with pathological complete response to neoadjuvant chemotherapy. Breast Cancer Research and Treatment 2014;148(3):525-34

[25] Wimberly H, Brown JR, Schalper K, Haack H, Silver MR, Nixon C, et al. PD-L1 Expression Correlates with Tumor-Infiltrating Lymphocytes and Response to Neoadjuvant Chemotherapy in Breast Cancer. Cancer Immunology Research 2015;3(4):326-32.

[26] Sorensen SF, Zhou W, Dolled-Filhart M, Georgsen JB, Wang Z, Emancipator K, et al. PD-L1 Expression and Survival among Patients with Advanced Non-Small Cell Lung Cancer Treated with Chemotherapy. Translational Oncology 2016;9(1):64-9.

[27] Horn T, Laus J, Seitz AK, Maurer T, Schmid SC, Wolf P, et al. The prognostic effect of tumour-infiltrating lymphocytic subpopulations in bladder cancer. World Journal of Urology 2016;34(2):181-7.

[28] Sharma P, Shen Y, Wen S, Yamada S, Jungbluth AA, Gnjatic $\mathrm{S}$, et al. CD8 tumor-infiltrating lymphocytes are predictive of survival in muscle-invasive urothelial carcinoma. Proc Natl Acad Sci U S A 2007;104(10):3967-72. 